Reihe Ökonomie Economics Series

\title{
Growth and the Ageing Joneses
}

Walter H. Fisher, Ben J. Heijdra 

Reihe Ökonomie Economics Series

\section{Growth and the Ageing Joneses}

Walter H. Fisher, Ben J. Heijdra

November 2008

Institut für Höhere Studien (IHS), Wien

Institute for Advanced Studies, Vienna 


\section{Contact:}

Walter H. Fisher

Department of Economics and Finance

Institute for Advanced Studies

Stumpergasse 56

1060 Vienna, Austria

용: $+43 / 1 / 59991-253$

Fax: +43-159991-555

email: fisher@ihs.ac.at

Ben J. Heijdra

Department of Economics

University of Groningen

P.O. Box 800

9700 AV Groningen, The Netherlands

email: b.j.heijdra@heijdra.org

and

Institute for Advanced Studies, Netspar, CESifo

Founded in 1963 by two prominent Austrians living in exile - the sociologist Paul F. Lazarsfeld and the economist Oskar Morgenstern - with the financial support from the Ford Foundation, the Austrian Federal Ministry of Education and the City of Vienna, the Institute for Advanced Studies (IHS) is the first institution for postgraduate education and research in economics and the social sciences in Austria. The Economics Series presents research done at the Department of Economics and Finance and aims to share "work in progress" in a timely way before formal publication. As usual, authors bear full responsibility for the content of their contributions.

Das Institut für Höhere Studien (IHS) wurde im Jahr 1963 von zwei prominenten Exilösterreichern dem Soziologen Paul F. Lazarsfeld und dem Ökonomen Oskar Morgenstern - mit Hilfe der FordStiftung, des Österreichischen Bundesministeriums für Unterricht und der Stadt Wien gegründet und ist somit die erste nachuniversitäre Lehr- und Forschungsstätte für die Sozial- und Wirtschaftswissenschaften in Österreich. Die Reihe Ökonomie bietet Einblick in die Forschungsarbeit der Abteilung für Ökonomie und Finanzwirtschaft und verfolgt das Ziel, abteilungsinterne Diskussionsbeiträge einer breiteren fachinternen Öffentlichkeit zugänglich zu machen. Die inhaltliche Verantwortung für die veröffentlichten Beiträge liegt bei den Autoren und Autorinnen. 


\section{Abstract}

We incorporate Keeping-up-with-the-Joneses (KUJ) preferences into the Blanchard-Yaari (BY) framework and develop, using an $A K$ technology, a model of balanced growth. In this context we investigate status preference, demographic, and pension policy shocks. We find that a higher degree of KUJ lowers economic growth, while, in contrast, a decrease in the fertility and mortality rates increase it. In the second part of the paper we extend the model by incorporating a Pay-as-you-go (PAYG) pension system with a statutory retirement date. This introduces a life-cycle in human wealth earnings and implies that the growth rate is higher under PAYG. We also consider the implications of an increase in the retirement date under both defined benefit and defined contribution schemes.

\section{Keywords}

Relative consumption, OLG, endogenous growth, pension reform

\section{JEL Classification}

D91, E21, H55 


\section{Comments}

The authors would like to thanks Jochen Mierau and Ward Romp for helpful comments. 


\section{Contents}

1 Introduction 1

2 The Macroeconomy 3

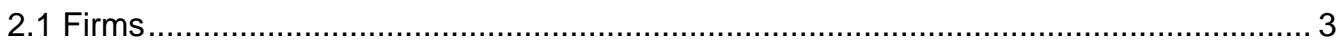

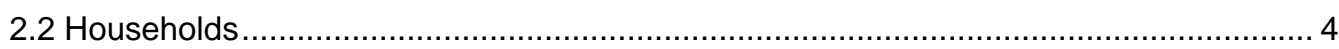

3 Aggregation and the Macroeconomic Equilibrium 5

4 Steady-State Growth 7

5 Comparative Static Effects 9

6 Introducing a PAYG Pension System 11

7 Pension Policy and Economic Growth 14

8 Conclusions 17

Appendix A: Derivation of equations (16)-(17) 19

Appendix B: Conditions on steady-state profiles $\quad 20$

Appendix C: Derivation of (46) and (47) 22

Appendix D: PAYG and the welfare of newborns 23

$\begin{array}{ll}\text { References } & 23\end{array}$ 



\section{Introduction}

Since the 1980s one of the main streams of research investigating the conditions for sustained economic growth has viewed the accumulation of capital, broadly measured, as one of its key sources. This approach, often termed the " $A K$ " framework, has been propagated, among others, by authors such as Romer (1986), Barro (1990), and Rebelo (1991). In its canonical, single-factor form, the $A K$ model yields a constant balanced growth rate with no transitional dynamics. In contrast to the standard Solow model, tax and government infrastructure policy (see Barro, 1990) affects the growth rate by changing the rate of return on capital.

The studies cited above all employ the representative agent (RA) framework. In this paper we seek to extend the insights of the $A K$ setting by adopting the overlapping generations (OLG) approach to specifying the consumer sector. Specifically, we use the Blanchard (1985)Yaari (1965) continuous-time framework to model the decisions of finite-lived consumers. A central characteristic of the Blanchard-Yaari (BY) model is the demographic turnover from old to young population cohorts. Since the asset-poor young replace the asset-rich old in this setting, demographic turnover influences the economy's saving and, thus, its accumulation of capital. A key advantage, then, of the BY framework is that it enables us to consider how demographic parameters influence economic growth in the $A K$ context in which capital accumulation plays the decisive role. Since population dynamics obviously depends on factors such as birth and mortality rates, we can use our model to investigate how demographic shocks affect the balanced growth rate. ${ }^{1}$ Due to its well-defined population dynamics, we can use the BY model to investigate the effects of public policies, in particular, Pay-as-you-go (PAYG) pensions, that have a significant intergenerational component.

Another important property of the BY framework, as recently shown by Fisher and Heij dra (2008) in an exogenous growth setting, is that the importance of demographic turnover also depends on agents' preferences, specifically on their attitude to status. In our endogenous growth framework, this allows us to ask the question whether or not status competition is an engine of economic growth. Recent authors who investigate this issue using an endogenous growth, representative agent (RA) framework that specifies consumption as the reference good include Alvarez-Cuadrado et al. (2004), Liu and Turnovsky (2005), and Turnovsky and Monteiro (2007). The specification used by these authors is often termed the "Keeping up with the Joneses" (KUJ) specification of status preferences. ${ }^{2}$ This literature does

\footnotetext{
${ }^{1}$ In non-endogenous growth contexts, recent authors who employ the BY framework to consider the effects of demographic shocks include Heijdra and Ligthart (2006) and Bettendorf and Heijdra (2006), the latter employing a small open economy framework. In this research demographic shocks are time-dependent, though cohort independent, an approach we follow here.

${ }^{2}$ Strictly speaking, the Alvarez-Cuadrado et al. (2004) and Turnovsky and Monteiro (2007) employ a "Catch-
} 
not, however, deliver an unambiguous answer regarding whether or not status competition is growth-promoting. Indeed, the relationship between status and growth in this context is highly sensitive to the specification of preferences and technology. ${ }^{3}$

The RA model is, moreover, a restrictive one to analyze the implications of status preferences, since all agents end up with the same consumption and asset holdings in the symmetric equilibrium, a situation implying that no one wins the "rat race". In contrast, agents of different ages, or "vintages", in the OLG framework possess distinct stocks of wealth and enjoy distinct levels of consumption. An economy-wide shift in KUJ then has age-dependent effects in the OLG context. A framework in which differences among individuals persist over time is, we believe, a promising avenue to explore the macroeconomic implications of status competition. Another important task in this paper, then, is to extend the findings of Fisher and Heijdra (2008) to the endogenous growth context and to consider the implications of changes in the degree of status preference.

Among our results, we show that the balanced growth rate, due to intergenerational turnover in financial wealth, is lower in the BY framework compared to its RA counterpart. We also consider demographic disturbances characteristic of advanced societies; falls in fertility and rises in longevity. In this context we find that while a decline in fertility and a rise in longevity both increase the growth rate, they have opposite implications for the consumption-capital ratio: the latter rises in response to a "baby bust" and falls subsequent to a jump in life-expectancy. Furthermore, we show that an increase in the degree of status preference lowers economic growth, since generational turnover, which tends to reduce growth, becomes more important in this scenario. Finally, in the second part of the paper, we investigate the implications of incorporating a PAYG pension system, featuring an exogenous retirement date. We find that economic growth is higher, given our design of the scheme, under PAYG. Again, it is demographic turnover that provides the source for this result. Specifically, PAYG pensions introduce a 'wedge' between the human wealth of newborns and the average stock of human wealth. Since newborns possess more human wealth than older agents under the PAYG system, the intergenerational turnover in human wealth provides a countervailing element to the turnover in financial wealth, which, as indicated, lowers economic growth in the BY framework.

ing up with the Joneses" approach in which reference consumption depends on past consumption and evolves over time.

${ }^{3}$ Alvarez-Cuadrado et al. (2004) find that the role of reference consumption in determining the response to macroeconomic shocks depends on whether $A K$ or the more flexible Cobb-Douglas technology is assumed. In Liu and Turnovsky (2005) the effect of KUJ on balanced growth is a function of the intertemporal elasticity of substititution. Turnovsky and Monteiro (2007) show that consumption externalities affect the long-run equilibrium if and only if work effort is endogenous. 
The paper is organized as follows. Section 2 outlines the firm and household sectors of the economy, which are aggregated in section 3 to determine the macroeconomic equilibrium. Section 4 derives the balanced rate of endogenous growth, while section 5 investigates how the latter is influenced by demographic and status preference shocks. Section 6 introduces the PAYG system into our OLG framework, which we analyze in section 7. There, we consider, first, the effect of public pensions on the rate of growth and, second, how an increase in the statutory retirement age influences the growth rate in both defined benefit and defined contribution schemes. We close in section 8 with brief concluding remarks and include an appendix containing supporting mathematical results.

\section{The Macroeconomy}

\subsection{Firms}

We begin by first analyzing the economy's firm sector. This permits us to describe the engine of endogenous growth, which relies, in the spirit of Romer (1989) and Saint-Paul (1992), on an inter-firm externality. The latter also leads to a constant (real) interest rate, a result that simplifies the derivation of the macroeconomic equilibrium. The firm sector is made up of a large number of perfectly competitive firms producing a homogenous good. At the individual firm level, output technology is Cobb-Douglas:

$$
Y_{i}(t)=F\left[K_{i}(t), L_{i}(t)\right] \equiv Z(t) \cdot K_{i}(t)^{\varepsilon} L_{i}(t)^{1-\varepsilon}, \quad 0<\varepsilon<1,
$$

where $Y_{i}(t)$ represents net output ${ }^{4}$ of firm $i, K_{i}(t)$ is the capital stock, $L_{i}(t)$ is labor supply (coinciding here with the population), and $Z(t)$ is total factor productivity common to all firms. For simplicity, we assume that capital accumulation does not incur adjustment costs. As usual under profit maximization, the rental values of capital and labor correspond to their marginal physical products:

$$
w(t)=\frac{\partial Y_{i}(t)}{\partial L_{i}(t)}=(1-\varepsilon) Z(t) \cdot k_{i}(t)^{\varepsilon}, \quad r(t)=\frac{\partial Y_{i}(t)}{\partial K_{i}(t)}=\varepsilon Z(t) \cdot k_{i}(t)^{\varepsilon-1},
$$

where $k_{i}(t) \equiv K_{i}(t) / L_{i}(t)$ is the capital-labor ratio. Moreover, since each firm faces the same wage rate and rental rate of capital, each has the same capital-labor ratio, $k_{i}(t)=k(t)$, which implies that firm output corresponds to $Y_{i}(t)=Z(t) L_{i}(t) k^{\varepsilon}(t)$. To obtain economy-wide relationships, we define: $Y(t) \equiv \sum_{i} Y_{i}(t), K(t) \equiv \sum_{i} K_{i}(t)$, and $L(t) \equiv \sum_{i} L_{i}(t)$. In turn, the inter-firm externality is given by:

$$
Z(t)=Z_{0} \cdot k(t)^{1-\varepsilon}, \quad Z_{0}>0,
$$

\footnotetext{
${ }^{4}$ That is, net output incorporates capital stock depreciation
} 
which implies that individual firms in this setting benefit from a rise in the average capital intensity. Aggregating firm output and substituting for $Z(t)=Z_{0} \cdot k(t)^{1-\varepsilon}$, we obtain the linear-in-capital, economy-wide production function $Y(t)=Z_{0} K(t)$. Substituting $Z(t)=$ $Z_{0} \cdot k(t)^{1-\varepsilon}$ into the marginal productivity conditions (2), we calculate expressions for the wage and the interest rate:

$$
w(t)=(1-\varepsilon) y(t)=(1-\varepsilon) Z_{0} k(t), \quad r(t)=r=\varepsilon Z_{0}>0 .
$$

Clearly, the interest rate is a positive constant, a result that is the source of continued growth. In contrast, agents can look forward to ongoing wage growth in this setting.

\subsection{Households}

We assume that the economy consists of agents of different birth dates, or "vintages", who compare their own consumption $\bar{c}(v, \tau)$ to the average level of consumption $c(\tau)$. Following Fisher and Heijdra (2008), for a consumer born at time $v(v \leq t)$ lifetime utility at $t$ equals:

$$
\Lambda(v, t)=\int_{t}^{\infty} \ln \bar{x}(v, \tau) e^{(\rho+\beta)(t-\tau)} d \tau,
$$

where $\rho$ is the rate of time preference, $\beta$ is the given instantaneous death probability (independent of age), and $\bar{x}(v, \tau)$ is the instantaneous subfelicity function defined as:

$$
\bar{x}(v, \tau) \equiv \frac{\bar{c}(v, \tau)-\alpha c(\tau)}{1-\alpha}, \quad \alpha<1,
$$

where the parameter $\alpha$ determines the agent's attitude to status competition. If $0<\alpha<1$, agents exhibit jealousy of the consumption of others. On the other hand, if $\alpha<0$, then agents express admiration for the consumption of others. The preferences in (6) satisfy the conditions for "Keeping up with the Joneses" (KUJ). ${ }^{5}$

The budget identity of an agent born at time $v$ equals:

$$
\dot{\bar{a}}(v, \tau)=(r+\beta) \bar{a}(v, \tau)+w(\tau)-\bar{c}(v, \tau),
$$

where $\bar{a}(v, \tau)$ represents assets, $r$ is the fixed interest rate, and $w(\tau)$ is the cohort-independent wage rate earned by agents who supply one unit of work effort. Assets yield an annuity income of $(r+\beta) \bar{a}(v, \tau)$, which consists of interest payments $r \bar{a}(v, \tau)$ and annuity receipts $\beta \bar{a}(v, \tau)$. Employing the usual methods of optimal control, we calculate the following timeprofile for $\bar{x}(v, \tau)$ :

$$
\frac{\dot{\bar{x}}(v, \tau)}{\bar{x}(v, \tau)}=r-\rho, \quad r>\rho .
$$

\footnotetext{
${ }^{5} \mathrm{KUJ}$ is satisfied with $U[\cdot] \equiv \ln x(v, \tau)$, since $\partial^{2} U[\cdot] / \partial \bar{c} \partial c=c /(c-\alpha \bar{c})^{2}>0$. See Dupor and Liu (2003) and Liu and Turnovsky (2005) for a detailed characterization of relative consumption preferences.
} 
The necessary condition $r>\rho$ implies that we focus on a given rising profile of $\bar{x}(v, \tau)$. In (8) we also obtain the usual BY-result that the probability of death $\beta$ cancels out along individual time-profiles, since the (higher) annuity rate of return $r+\beta$ is offset by the (greater) effective rate of time preference $\rho+\beta .^{6}$ In fact, at the aggregate level, the crucial demographic parameter (see (16) below) is the fertility rate, denoted by $\eta$.

The next step is to calculate the intertemporal budget constraint of the individual. Integrating (7) subject to the NPG condition $\lim _{\tau \rightarrow \infty} \bar{a}(v, \tau) e^{(r+\beta)(t-\tau)}=0$, yields:

$$
\int_{t}^{\infty}[(1-\alpha) \bar{x}(v, \tau)+\alpha c(\tau)] e^{(r+\beta)(t-\tau)} d \tau=\bar{a}(v, t)+h(t),
$$

where $h(t)=\int_{t}^{\infty} w(\tau) e^{(r+\beta)(t-\tau)} d \tau$ is age-independent human wealth. ${ }^{7}$ Equation (9) states that the present discounted value of a weighted average of individual subfelicity and average consumption - where the weights depend on the parameter $\alpha$ - corresponds to the aggregate of the agent's financial and human wealth. Integrating (8) to obtain $\bar{x}(v, \tau)=$ $\bar{x}(v, t) e^{(r+\beta)(\tau-t)}, \tau \geq t$, we can show that (9) reduces to:

$$
(1-\alpha) \frac{\bar{x}(v, t)}{\rho+\beta}=\bar{a}(v, t)+h(t)-\alpha \Gamma(t),
$$

where $\Gamma(t) \equiv \int_{t}^{\infty} c(\tau) e^{(r+\beta)(t-\tau)} d \tau$. Substitution of $\bar{x}(v, t)$ from (6) in (10), yields an expression for individual consumption that is a function of average consumption as well as wealth:

$$
\bar{c}(v, t)=(\rho+\beta)[\bar{a}(v, t)+h(t)]+\alpha[c(t)-(\rho+\beta) \Gamma(t)] .
$$

Among the features that emerge from (11) is that individual consumption depends on average consumption, due to the existence of a consumption externality, i.e., $\alpha \neq 0$. Otherwise, an agent consumes - as in the standard setting — out of his wealth according to $\rho+\beta$, the marginal propensity to consume.

\section{Aggregation and the Macroeconomic Equilibrium}

In this section of the paper we first specify the economy's demography. This is necessary to aggregate the individual relationships and, thus, to describe the OLG macroeconomy. Letting $\eta$ represent the birth rate, the (constant) population growth rate is $n \equiv \eta-\beta$, with $\beta$,

\footnotetext{
${ }^{6}$ Neverthless, the level of an agent's consumption does depend on $\beta$.

${ }^{7}$ Observe that in a growth context in which wages follow the path $w(\tau)=w(t) e^{\hat{\gamma}(\tau-t)}$ (where the growth rate $\hat{\gamma}$ is determined in section 4), human wealth depends on time, $t$, but not on the agent's age, $t-v$. In contrast, human wealth will be both time- and age-dependent once we introduce a PAYG system in section 6 below.
} 
as indicated, the mortality probability. Through time, individual population cohorts $L(v, t)$ shrink as their members die off. The population proportion of cohort $v$ at time $t$ thus equals:

$$
l(v, t) \equiv \frac{L(v, t)}{L(t)}=\eta e^{\eta(v-t)}, \quad t \geq v
$$

which enables us to define the per-capita average values of consumption and financial assets:

$$
c(t) \equiv \int_{-\infty}^{t} l(v, t) \bar{c}(v, t) d v, \quad a(t) \equiv \int_{-\infty}^{t} l(v, t) \bar{a}(v, t) d v,
$$

where $c(t)$ represents, furthermore, the consumption externality from the individual's point of view. Aggregating individual consumption (11), we obtain:

$$
c(t)=(\rho+\beta)[a(t)+h(t)]+\alpha[c(t)-(\rho+\beta) \Gamma(t)] .
$$

Subtracting (14) from (11), we find:

$$
\bar{c}(v, t)-c(t)=(\rho+\beta)[\bar{a}(v, t)-a(t)]
$$

where the difference between individual and average consumption depends on the difference between individual and average financial wealth, a fact we use below to draw the distinctions between the BY and RA frameworks.

The key step to derive the growth equilibrium is to obtain the differential equations for average consumption and financial assets, $\dot{c}(t)$ and $\dot{a}(t)$. The details of this exercise are given in Appendix A. Using the expressions for $\dot{c}(t)$ and $\dot{a}(t)$, the rate of return and aggregate relationships of the production sector, and the fact that only physical capital is used for savings, $k(t) \equiv a(t)$, we derive the following macroeconomic equilibrium:

$$
\begin{aligned}
& \frac{\dot{c}(t)}{c(t)}=r-\rho-\frac{\eta(\rho+\beta)}{1-\alpha} \cdot \frac{k(t)}{c(t)}, \\
& \dot{k}(t)=[r-n] k(t)+w(t)-c(t), \\
& w(t)=(1-\varepsilon) y(t), \quad r=\varepsilon Z_{0}, \\
& y(t)=Z_{0} k(t) .
\end{aligned}
$$

The dynamics of consumption is described by (16), while (17) governs the accumulation of physical capital, $n \equiv \eta-\beta$. Equation (18) reiterates the expressions for the wage and the interest rate, while (19) is the per-capita version of the production function. In contrast to equations (17)-(19), which emerge in the usual Ramsey framework, equation (16) for consumption dynamics merits additional comment. The third term on the right-hand-side of (16) is typical in the BY-setting and represents the effect that demographic turnover has on 
consumption dynamics and, as we show below, economic growth. To see this, we evaluate (15) at $v=t$ and impose $k(t) \equiv a(t)$. This yields $c(t)-\bar{c}(t, t)=(\rho+\beta) k(t)$, which, if substituted in (16), results in the following alternative representation of $\dot{c}(t) / c(t)$ :

$$
\frac{\dot{c}(t)}{c(t)}=r-\rho-\frac{\eta}{1-\alpha} \cdot \frac{c(t)-c(t, t)}{c(t)} .
$$

The term $[c(t)-\bar{c}(t, t)]$, corresponding to the difference between average and new-born consumption, measures the effect of intergenerational turnover. In the BY-framework older generations are replaced by newborns. Because, however, agents are born with no financial wealth, their consumption levels fall short of that of their older counterparts. Consequently, the replacement of asset-rich by asset-poor population cohorts reduces the growth rate of average consumption. This is the case even though the growth rate of individual consumption, $\dot{\bar{c}}(v, \tau) / \bar{c}(v, \tau)$, is the same for each generation facing the given interest rate $r$.

\section{Steady-State Growth}

In this model the single accumulable factor of production, physical capital, has the constant returns to scale property. Consequently, the long-run equilibrium is characterized by a sustained, balanced growth rate, denoted by $\hat{\gamma}$. Furthermore, the economy exhibits no transitional dynamics. To see why this is the case, let $x(t) \equiv c(t) / k(t)$ represent the consumptioncapital ratio and employ (16)-(19) to derive $\dot{x}(t) / x(t)$ :

$$
\frac{\dot{x}(t)}{x(t)}=\left[r-\rho+Z_{0}-n\right]+x(t)-\frac{\eta(\rho+\beta)}{1-\alpha} \cdot \frac{1}{x(t)} .
$$

It is straightforward to show that (21) is an unstable differential equation. Consequently, a stable equilibrium is achieved only if the consumption-capital ratio attains a constant value, $x(t) \equiv \hat{x}, \forall t \geq 0$, which, in turn, implies that the economy grows at the rate $\hat{\gamma}$ through time. The resulting steady-state growth profiles of capital, wages, and consumption are $\hat{k}(t)=\hat{k}_{0} e^{\hat{\gamma} t}, \hat{w}(0)=\hat{w}_{0} e^{\hat{\gamma} t}$, and $\hat{c}(t)=\hat{c}_{0} e^{\hat{\gamma} t}$, where $\hat{k}(0)=\hat{k}_{0}, \hat{w}(0)=\hat{w}_{0}$, and $\hat{c}(0)=\hat{c}_{0}$ denote their respective initial values.

To determine the solution for the balanced growth rate, we evaluate (16)-(17) along the steady-state profile:

$$
\hat{\gamma}=r-\rho-\frac{\eta(\rho+\beta)}{1-\alpha} \frac{1}{\hat{x}^{\prime}}, \quad \hat{\gamma}=r+(1-\varepsilon) Z_{0}-n-\hat{x},
$$

where $\hat{x} \equiv \hat{c} / \hat{k}$ is the consumption-capital ratio. To further simplify the problem, we define the growth-adjusted interest rate as $\hat{r}_{g} \equiv r-\hat{\gamma}$ and re-express (22) as:

$$
\left(\hat{r}_{g}-\rho\right) \hat{x}=\frac{\eta(\rho+\beta)}{1-\alpha}, \quad \hat{x}=\hat{r}_{g}+(1-\varepsilon) Z_{0}-n .
$$




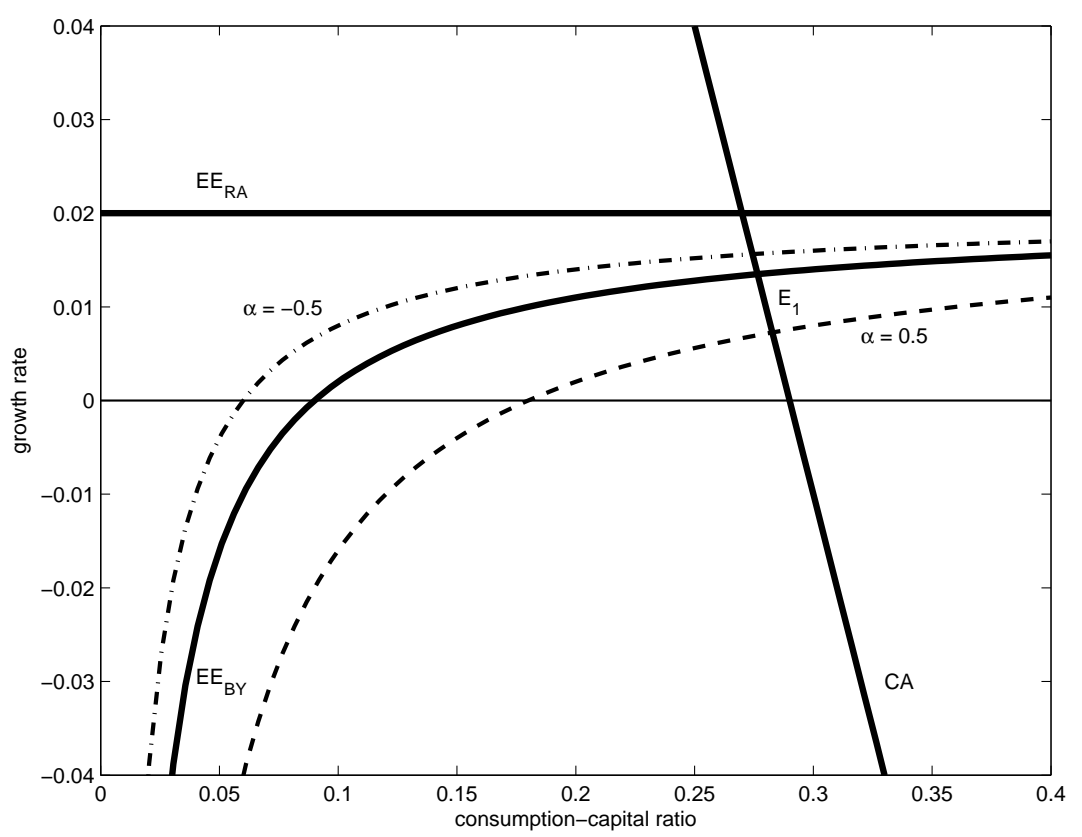

Figure 1: Growth and the KUJ effect

Combining the relationships in (23), we form the polynomial $\Phi(s)$ :

$$
\Phi(s) \equiv(s-\rho) \cdot\left[s+(1-\varepsilon) Z_{0}-n\right]-\frac{\eta(\rho+\beta)}{1-\alpha},
$$

where $\Phi\left(\hat{r}_{g}\right)=0$ solves for the growth-adjusted interest rate $\hat{r}_{g}$. There is only one feasible solution with $\hat{x}>0$. This is satisfied with $\hat{r}_{g} \equiv r-\hat{\gamma}>\rho$ and $\hat{r}_{g}>n-(1-\varepsilon) Z_{0} \cdot{ }^{8}$ The Euler and market clearing relationships can also be combined to determine the polynomial $\Gamma(s)$ that solves for the consumption-capital ratio, i.e., $\Gamma(\hat{x})=0$ :

$$
\Gamma(s) \equiv s^{2}-\left[\rho+(1-\varepsilon) Z_{0}-n\right] s-\frac{\eta(\rho+\beta)}{1-\alpha} .
$$

Using (22), we illustrate in Figure 1 the OLG balanced growth equilibrium. The positivelysloped locus $\mathrm{EE}_{\mathrm{BY}}$ represents the Euler equation, while the downward-sloping line CA depicts market clearing. The relationships (both solid) have the following slopes:

$$
\left.\frac{d \hat{\gamma}}{d \hat{x}}\right|_{\mathrm{EE}_{\mathrm{BY}}}=-\frac{\eta(\rho+\beta)}{(1-\alpha) \hat{x}^{2}}>0,\left.\quad \frac{d \hat{\gamma}}{d \hat{x}}\right|_{\mathrm{CA}}=-1 .
$$

\footnotetext{
${ }^{8}$ In Appendix B we derive the conditions for a feasible solution of the steady-state growth profile. In particular, we show that $\hat{r}_{g}>\rho$ is necessary for $\bar{k}(0, t)>0$. We also determine the necessary conditions for $\bar{c}(0, t)>0$, along with the upper and lower bounds on the status parameter $\alpha$.
} 
The intersection of $\mathrm{EE}_{\mathrm{BY}}$ and $\mathrm{CA}$ at point $\mathrm{E}_{1}$ illustrates the OLG solution $\left(\hat{x}_{1}, \hat{\gamma}_{1}\right)$ determined by (24)-(25). The positively-sloped $\mathrm{EE}_{\mathrm{BY}}$ locus reflects the fact that the higher is the consumption-capital ratio $\hat{x}$, the weaker is the intergenerational turnover effect, which implies a greater growth rate. Along the negatively-sloped CA line, higher values of $\hat{x}$ translate directly into lower rates of growth $\hat{\gamma}$. In addition, we depict in Figure 1 the solid horizontal line $\mathrm{EE}_{\mathrm{RA}}$ representing the Euler equation for the RA case. ${ }^{9}$ Observe that it lies uniformly above its $\mathrm{EE}_{\mathrm{BY}}$ counterpart. The growth rate in the RA economy is simply the difference between the fixed interest rate and the given rate of time preference, $r-\rho$ (so that $\hat{r}_{g}=\rho$ ). The relationships in Figure 1 are generated using a numerical solution of the model that assumes the structural parameters take the following values:

$$
\varepsilon=0.20, \quad r=0.06, \quad \rho=0.04, \quad \alpha=0, \quad \beta=0.02, \quad \eta=0.03 .
$$

Clearly, the balanced growth rate $\hat{\gamma}$ is lower in the BY case compared to its RA counterpart, while the consumption-capital ratio $\hat{x}$ is higher. Indeed, while the balanced growth rate in the RA economy depends only on technology and the pure of rate of time preference, in the BY setting it is also a function of agents' attitude to status, parameterized by $\alpha$, as well as demographic parameters, $\eta$ and $\beta$, that reflect intergenerational turnover. We next employ our OLG equilibrium to investigate the effect of changes in agents' attitude to consumption externalities and one-time demographic shocks.

\section{Comparative Static Effects}

To determine the effects of demographic and status preference shocks on the OLG growth rate, we evaluate (24)-(25) at the solution values $\left(\hat{x}, \hat{r}_{g}\right)$ :

$$
\begin{aligned}
\Phi\left(\hat{r}_{g}, \eta, \beta, \alpha\right) & \equiv\left(\hat{r}_{g}-\rho\right) \cdot\left[\hat{r}_{g}+(1-\varepsilon) Z_{0}-(\eta-\beta)\right]-\frac{\eta(\rho+\beta)}{1-\alpha} \equiv 0, \\
\Gamma(\hat{x}, \eta, \beta, \alpha) & =\hat{x}^{2}-\left[\rho+(1-\varepsilon) Z_{0}-(\eta-\beta)\right] \hat{x}-\frac{\eta(\rho+\beta)}{1-\alpha} \equiv 0 .
\end{aligned}
$$

In all instances, the economy jumps immediately its new steady-state growth path. We consider first the consequences of an increase in jealousy (i.e., $\alpha$ rises from 0 to 0.5). Using (27), it is straightforward to show:

$$
\begin{aligned}
\frac{\partial \hat{r}_{g}}{\partial \alpha} & =-\frac{\partial \hat{\gamma}}{\partial \alpha}=-\frac{\partial \Phi\left(\hat{r}_{g}, \eta, \beta, \alpha\right) / \partial \alpha}{\partial \Phi\left(\hat{r}_{g}, \eta, \beta, \alpha\right) / \partial \hat{r}_{g}}>0 \\
\frac{d \hat{x}}{d \alpha} & =-\frac{\partial \Gamma(\hat{x}, \eta, \beta, \alpha) / \partial \alpha}{\partial \Gamma(\hat{x}, \eta, \beta, \alpha) / \partial \hat{x}}>0
\end{aligned}
$$

\footnotetext{
${ }^{9}$ The expressions for the RA version of the model with population growth are obtained by setting $\eta=0$ (no new disconnected agents enter the economy) and $\beta=-n$ (population growth consists of the arrival of new family members).
} 
where the signs in (28) imply that a rise in $\alpha$ causes a decline in the growth rate and an increase in the consumption-capital ratio. The larger is the status externality, the more important is intergenerational turnover, which implies that average consumption rises at the expense of saving, leading to a permanent fall in $\hat{\gamma}$ and rise in $\hat{x}$. In terms of Figure 1 , the increase in $\alpha$ causes the $\mathrm{EE}_{\mathrm{BY}}$ to shift down (CA is unaffected), leading to the new equilibrium featuring a lower value of $\hat{\gamma}$ and an increase in $\hat{x}$. This is the endogenous growth analogue of the result of Fisher and Heijdra (2008), showing that a rise in status preference leads in steady state to a decline in the stock of capital and a rise in consumption. The distinction is that here adjustment takes place instantly, while the Fisher and Heijdra (2008) findings feature an initial increase in consumption, followed by a continuous decline in its level, accompanied by a reduction in the capital stock. For comparison, observe that we also depict in Figure 1 the case of admiration, i.e., a fall in $\alpha$ from 0 to -0.5 , causing $\mathrm{EE}_{\mathrm{BY}}$ to shift-up and resulting in a rise in $\hat{\gamma}$ and a fall in $\hat{x}$.

Considering next the case of a baby bust ( $\eta$ falls from 0.03 to 0.02 ), differentiation of (27) with respect to $\eta$ yields:

$$
\begin{aligned}
\frac{\partial \hat{r}_{g}}{\partial \eta} & =-\frac{\partial \hat{\gamma}}{\partial \eta}=-\frac{\partial \Phi\left(\hat{r}_{g}, \eta, \beta, \alpha\right) / \partial \eta}{\partial \Phi\left(\hat{r}_{g}, \eta, \beta, \alpha\right) / \partial \hat{r}_{g}}>0, \\
\frac{d \hat{x}}{d \eta} & =-\frac{\partial \Gamma(\hat{x}, \eta, \beta, \alpha) / \partial \eta}{\partial \Gamma(\hat{x}, \eta, \beta, \alpha) / \partial \hat{x}}<0,
\end{aligned}
$$

where the signs in (29) follow from $\hat{r}_{g}>\rho$ and $\hat{r}_{g}>n-(1-\varepsilon) Z_{0}$. According to (29), a decline in fertility, since it reduces the importance of intergenerational turnover, leads to an increase in economic growth and rise in the consumption-capital ratio. Graphically, this shock is illustrated in Figure 2, where the post-shock Euler and the market clearing relationships (dashed) result in a new equilibrium with higher values of $\hat{\gamma}$ and $\hat{x}$.

Finally, turning to the case of a longevity boom ( $\beta$ down from 0.02 to 0.01 ), we find: ${ }^{10}$

$$
\begin{aligned}
\frac{\partial \hat{r}_{g}}{\partial \beta} & =-\frac{\partial \hat{\gamma}}{\partial \beta}=-\frac{\partial \Phi\left(\hat{r}_{g}, \eta, \beta, \alpha\right) / \partial \eta}{\partial \Phi\left(\hat{r}_{g}, \eta, \beta, \alpha\right) / \partial \hat{r}_{g}}>0, \\
\frac{d \hat{x}}{d \beta} & =-\frac{\partial \Gamma(\hat{x}, \eta, \beta, \alpha) / \partial \beta}{\partial \Gamma(\hat{x}, \eta, \beta, \alpha) / \partial \hat{x}}>0,
\end{aligned}
$$

that this leads to a higher growth rate and - in contrast to a baby bust - a lower consumptioncapital ratio. Because agents live longer, they have the incentive to accelerate the accumulation of capital, increasing the balanced growth rate $\hat{\gamma}$. Since, however, this is spread-out over a longer lifetime, consumption falls relative to the stock of capital. We also illustrate this in Figure 2, which depicts the shift-up in $\mathrm{EE}_{\mathrm{BY}}$ and the shift-down in CA (dash-dotted) that leads to an increase in $\hat{\gamma}$ and a fall in $\hat{x}$.

\footnotetext{
${ }^{10}$ The sign of $\partial \hat{r}_{g} / \partial \beta$ follows from that of $\partial \Phi / \partial \beta$, which equals $\hat{r}_{g}-\rho-\eta /(1-\alpha)<0$. Since we can show $\hat{r}_{g}<\rho+\eta$ (see Appendix B), the latter holds whether or not $0<\alpha<1$ or $\alpha<0$.
} 


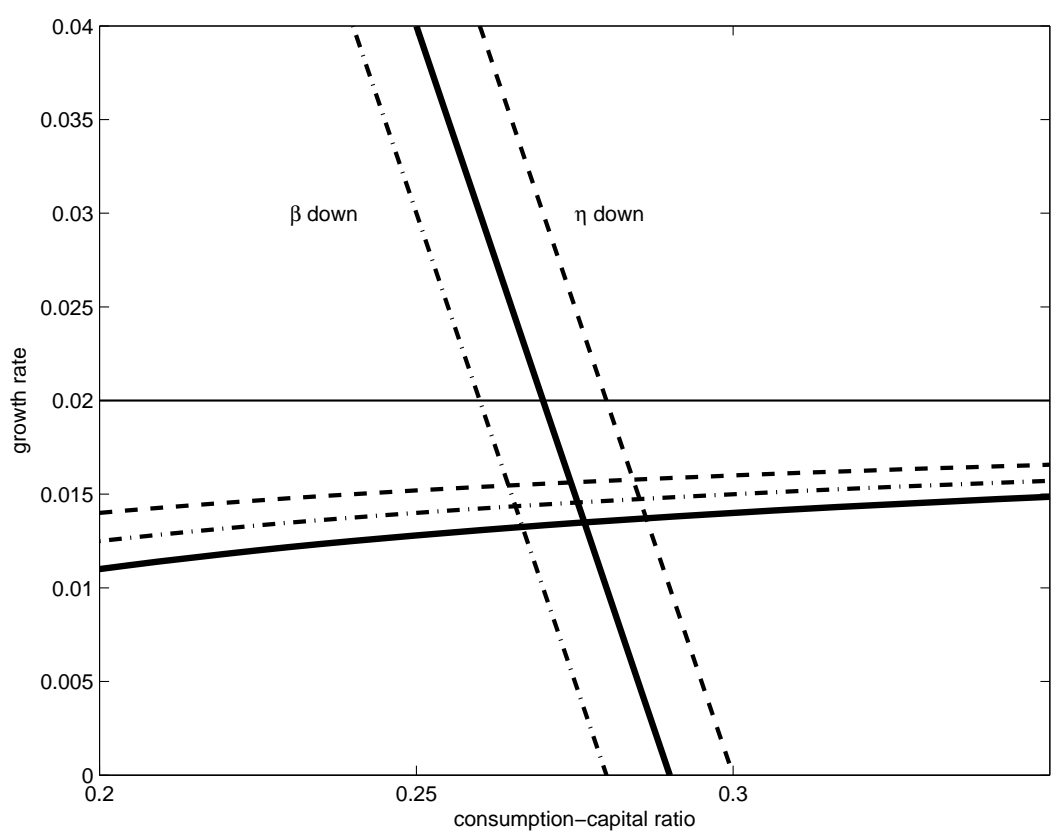

Figure 2: Growth and demographic shocks

\section{Introducing a PAYG Pension System}

We now extend the basic growth model to incorporate a PAYG pension system. Letting $\bar{z}(v, \tau)$ denote taxes (and transfers if negative), contributions are paid and benefits are received according the following scheme:

$$
\bar{z}(v, \tau)=\left\{\begin{array}{cl}
\theta \cdot w(\tau) & \text { for } \tau-v \leq u_{R} \\
-\pi \cdot w(\tau) & \text { for } \tau-v>u_{R}
\end{array},\right.
$$

where $\theta$ is the contribution rate, $\pi$ is the benefit rate (both indexed to the wage $w(\tau)$ ), and $u_{R}$ is the statutory retirement age. For realism, we assume that workers earn more than pensioners so that $1-\theta>\pi$. As in the benchmark specification, labor supply is exogenous, although modified to reflect mandatory retirement:

$$
\bar{n}(v, \tau)=\left\{\begin{array}{ll}
1 & \text { for } \tau-v \leq u_{R} \\
0 & \text { for } \tau-v>u_{R}
\end{array} .\right.
$$

According to (32), agents supply a "full" unit of labor until retirement after which they cease to work. This permits us to define the macroeconomic participation rate as:

$$
\frac{N(t)}{L(t)} \equiv \int_{-\infty}^{t} \bar{n}(v, t) l(v, t) d v=\int_{t-u_{R}}^{t} l(v, t) d v=1-e^{-\eta u_{R}},
$$


where $N(t)$ is the work force. Clearly, the participation rate rises with $u_{R}$, while a "baby bust" (decline in $\eta$ ) reduces it. This formulation allows us to define the dependency ratio as:

$$
d r \equiv d r\left(u_{R}, \eta\right)=\frac{e^{-\eta u_{R}}}{1-e^{-\eta u_{R}}}, \quad \frac{\partial d r}{\partial u_{R}}<0, \quad \frac{\partial d r}{\partial \eta}<0 .
$$

Not only does the PAYG system place a "wedge" between the workforce and the population, it also implies that an agent's human wealth is age-dependent. Letting $\bar{h}(v, \tau)$ represent individual human wealth, its weighted average equals:

$$
h(t) \equiv \int_{-\infty}^{t} l(v, t) \bar{h}(v, t) d v .
$$

Next, we impose sustainability of the PAYG system by assuming that contributions always equal payouts at all points in time:

$$
\int_{t-u_{R}}^{t} \theta w(t) L(v, t) d v=\int_{-\infty}^{t-u_{R}} \pi w(t) L(v, t) d v
$$

Substituting for $L(v, t)=L(t) \cdot l(v, \tau)$, using (12), the closure rule reduces to:

$$
\theta \cdot\left[1-e^{-\eta u_{R}}\right]=\pi \cdot e^{-\eta u_{R}},
$$

where one of $\theta, u_{R}$, and $\pi$ must be used to balance the PAYG budget. Observe that under a defined benefit (DB) scheme, $\pi$ and $u_{R}$ are held constant while $\theta$ balances the budget. In contrast, $\theta$ and $u_{R}$ are held constant while $\pi$ balances the budget under a defined contribution (DC) scheme. It is straightforward to show that the following relationships hold:

$$
1-\theta-\pi=\left\{\begin{array}{ll}
1-\frac{\pi}{1-e^{-\eta u_{R}}} & \text { DB } \\
1-\theta e^{\eta u_{R}} & \text { DC }
\end{array} .\right.
$$

It follows that:

$$
\frac{\partial(1-\theta-\pi)}{\partial u_{R}}>0 \quad(\text { for } \mathrm{DB}), \quad \frac{\partial(1-\theta-\pi)}{\partial u_{R}}<0 \quad \text { (for DC). }
$$

These results are important to investigate how a change in the statutory retirement date affects the balanced growth rate.

To solve the modified model, we follow the same procedure outlined above. The firm's problem is solved as in section 2.1, with $L_{i}(t)$ replaced by $N_{i}(t)$ in (1), and with $K(t) / N(t)$ affecting general productivity in (3). Since labor supply, according to (33), depends on the retirement date $u_{R}$, so does the wage rate $w(t)$ :

$$
w(t)=(1-\varepsilon) \frac{Y(t)}{N(t)}=(1-\varepsilon) \frac{Z_{0} k(t)}{1-e^{-\eta u_{R}}},
$$


where we substitute for $Y(t)=Z_{0} K(t), N(t) \equiv\left[1-e^{-\eta u_{R}}\right] L(t)$ and use $k(t) \equiv K(t) / L(t)$ to obtain (40). Observe that for a given value of $k(t)$, a later retirement date lowers the wage rate due to the expansion in labor supply.

Regarding the household's problem, we proceed along the same lines as above, with the exception that the agent's choices are made subject to (31). Consequently, we replace (7) by:

$$
\dot{\bar{a}}(v, \tau)=[r(\tau)+\beta] \bar{a}(v, \tau)+w(\tau)-\bar{c}(v, \tau)-\bar{z}(v, \tau) .
$$

Similarly, we replace $h(t)$ with $\bar{h}(v, t)$ in the expression (11) for individual consumption. In turn, an active agent possesses a human wealth level of:

$$
\begin{aligned}
\bar{h}(v, t) & \equiv \int_{t}^{v+u_{R}} w(\tau) e^{(r+\beta)(t-\tau)} d \tau-\int_{t}^{\infty} \bar{z}(v, \tau) e^{(r+\beta)(t-\tau)} d \tau \\
& =\int_{t}^{v+u_{R}}(1-\theta) w(\tau) e^{(r+\beta)(t-\tau)} d \tau+\int_{v+u_{R}}^{\infty} \pi w(\tau) e^{(r+\beta)(t-\tau)} d \tau,
\end{aligned}
$$

where we use (31) to obtain the second equality of (42). Substituting the path of wages in (42), $w(\tau)=w(t) \cdot e^{\hat{\gamma}(\tau-t)}, \tau \geq t$ (with $\hat{\gamma}$ determined in equilibrium), a worker's human wealth simplifies to: ${ }^{11}$

$$
\bar{h}(v, t)=\frac{w(t)}{r_{g}+\beta} \cdot\left[(1-\theta) \cdot\left[1-e^{\left(\hat{r}_{g}+\beta\right)\left(t-v-u_{R}\right)}\right]+\pi \cdot e^{\left(\hat{r}_{g}+\beta\right)\left(t-v-u_{R}\right)}\right], \quad t-v \leq u_{R} .
$$

Correspondingly, a retired person's human wealth is given by:

$$
\bar{h}(v, t)=\pi \int_{t}^{\infty} w(\tau) e^{(r+\beta)(t-\tau)} d \tau=\frac{\pi w(t)}{\hat{r}_{g}+\beta}, \quad t-v>u_{R} .
$$

To determine the economy's Euler equation, we use the method described in Appendix A for the standard formulation. It is straightforward to show that (16) becomes:

$$
\frac{\dot{c}(t)}{c(t)}=r(t)-\rho-\frac{\eta(\rho+\beta)}{1-\alpha} \cdot\left[\frac{k(t)}{c(t)}-\frac{\bar{h}(t, t)-h(t)}{c(t)}\right],
$$

where consumption dynamics now also depends on the intergenerational turnover, $[\bar{h}(t, t)-$ $h(t)]$, in human wealth. We show in Appendix C that $[\bar{h}(t, t)-h(t)]$ equals:

$$
\bar{h}(t, t)-h(t)=w(t) e^{-\beta u_{R}}(1-\theta-\pi) \cdot \frac{e^{-n u_{R}}-e^{-\hat{r}_{g} u_{R}}}{\hat{r}_{g}-n}>0,
$$

where $1-\theta>\pi \cdot{ }^{12}$ Clearly, agents are born with more human wealth than average, since they can look forward to the relatively longest period of high earnings. Moreover, the PAYG

\footnotetext{
${ }^{11}$ In the absence of a pension system, $\theta=\pi=0$ and $u_{R} \rightarrow \infty$ individual human wealth reduces to $\bar{h}(v, t)=$ $h(t)=w(t) /\left(r_{g}+\beta\right)$.

${ }^{12}$ Two further things are worth noting. First, the sign of (46) is guaranteed because $\frac{e^{-n u_{R}}-e^{-p_{g} u_{R}}}{\hat{r}_{g}-n}$ is positive regardless of the sign of $\hat{r}_{g}-n$. Second, under the Aaron condition, $\hat{r}_{g}>n$, it follows that for a given retirement age $u_{R}$, a newborn has a lower level of human wealth under the PAYG system than in the absence of such a system (see Appendix D).
} 
system affects the macroeconomy through the turnover in human wealth. Indeed, because newborn agents possess more human wealth than their older counterparts, this mitigates the fact that newborns are "asset poor" financially compared to older agents. This lessens the effects of intergenerational turnover in (45) and increases the growth in average consumption compared to an economy without a PAYG scheme, a result we prove subsequently. To sumup, PAYG macroeconomic equilibrium consists of $(45)$, where $[\bar{h}(t, t)-h(t)]$ is given by (46). The expressions for the interest rate are the same as stated in (18)-(19), while we replace the expression for the wage with (40). Finally, regarding market clearing, we replace (17) with: ${ }^{13}$

$$
\dot{k}(t)=[r-n] k(t)+w(t)\left(1-e^{-\eta u_{R}}\right)-c(t) .
$$

\section{Pension Policy and Economic Growth}

To investigate the implications of pension policy for economic growth, we first derive the modified economic dynamics. For the Euler relationship we substitute the equation for the wage $w(t)$ from (40) in that of $[\bar{h}(t, t)-h(t)]$ from (46) and use $y(t)=Z_{0} k(t)$. We then substitute the resulting expression in (45) to calculate:

$$
\frac{\dot{c}(t)}{c(t)}=r-\rho-\sigma \Omega\left(\hat{r}_{g}\right) \cdot \frac{k(t)}{c(t)}
$$

where $\hat{r}_{g} \equiv r-\hat{\gamma}, \sigma \equiv \eta(\rho+\beta) /(1-\alpha)$ is a positive constant, and:

$$
\Omega\left(\hat{r}_{g}\right) \equiv 1-\frac{(1-\varepsilon) r}{\varepsilon} \cdot d r\left(u_{R}\right) \cdot(1-\theta-\pi) \cdot \frac{1-e^{-\left(\hat{r}_{g}-n\right) u_{R}}}{\hat{r}_{g}-n}>0 .
$$

Observe that the difference between (48) and the Euler equation of the basic model (16) is that per capita consumption growth now depends on $\Omega\left(\hat{r}_{g}\right)$, which is itself a function of $\hat{\gamma}$ and incorporates features of the pension system. Similarly, combining (47) with (40) and $y(t)=Z_{0} k(t)$, the market clearing condition simplifies to:

$$
\dot{k}(t)=\left[r+(1-\varepsilon) Z_{0}-n\right] k(t)-c(t) .
$$

Evaluating (48)-(49) along the steady-state growth path $\hat{\gamma}$ and, as before, letting $\hat{x} \equiv \hat{c} / \hat{k}$, we obtain:

$$
\hat{\gamma}=r-\rho-\sigma \Omega(r-\hat{\gamma}) \cdot \frac{1}{\hat{x}^{\prime}}, \quad \hat{\gamma}=r+(1-\varepsilon) Z_{0}-\hat{x}-n .
$$

Observe that the expression for market clearing is identical to that from the basic model, implying that PAYG pensions affect the growth path only through the Euler relationship. To

\footnotetext{
${ }^{13}$ See Appendix $\mathrm{C}$ for the derivation of (47).
} 
distinguish the framework with public pensions from that of the basic framework, we let $\hat{r}_{g}^{P}$ $\left(\equiv r-\hat{\gamma}^{P}\right)$ and $\hat{x}^{P}$ represent, respectively, the growth-adjusted interest rate and consumptioncapital ratio under the PAYG plan. The system (50) becomes:

$$
\left(\hat{r}_{g}^{P}-\rho\right) \cdot \hat{x}^{P}=\sigma \Omega\left(\hat{r}_{g}^{P}\right), \quad \hat{x}^{P}=\hat{r}_{g}^{P}+(1-\varepsilon) Z_{0}-n
$$

Combining the expressions in (51), we obtain the polynomial determining $\hat{r}_{g}^{P}$ :

$$
\Phi\left(\hat{r}_{g}^{P}, \pi, \theta, u_{R}\right) \equiv\left(\hat{r}_{g}^{P}-\rho\right) \cdot\left[\hat{r}_{g}^{P}+(1-\varepsilon) Z_{0}-n\right]-\sigma \Omega\left(\hat{r}_{g}^{P}\right) \equiv 0,
$$

where we indicate in (52) that the solution depends on the parameters of the PAYG system. Equally, the polynomial solving for $\hat{x}^{P}$ corresponds to:

$$
\Gamma\left(\hat{x}^{P}, \pi, \theta, u_{R}\right)=\left(\hat{x}^{P}\right)^{2}-\left[\rho+(1-\varepsilon) Z_{0}-n\right] \hat{x}^{P}-\sigma \Omega\left(\hat{r}_{g}^{P}\right) \equiv 0 .
$$

We next show that the economy with PAYG pensions has a higher growth rate and a lower consumption-capital ratio than the economy lacking them. To do so, we linearize the polynomial $\Phi(s)$ given in (24) from the basic model about the PAYG equilibrium determined in (52). This yields:

$$
\left(\hat{r}_{g}^{P}-\rho\right) \cdot\left[\hat{r}_{g}^{P}+(1-\varepsilon) Z_{0}-n\right]-\sigma+\left[2 \hat{r}_{g}^{P}-(\rho+n)+(1-\varepsilon) Z_{0}\right] \cdot\left(\hat{r}_{g}-\hat{r}_{g}^{P}\right)=0 .
$$

Evaluating the first term in (54) at the PAYG equilibrium using (52), we solve for $\left(\hat{r}_{g}-\hat{r}_{g}^{P}\right)=$ $\left(\hat{\gamma}^{P}-\hat{\gamma}\right)$ :

$$
\hat{r}_{g}-\hat{r}_{g}^{P}=\hat{\gamma}^{P}-\hat{\gamma}=\frac{\sigma\left[1-\Omega\left(\hat{r}_{g}^{P}\right)\right]}{2 \hat{r}_{g}^{P}-(\rho+n)+(1-\varepsilon) Z_{0}}>0,
$$

which implies that the balanced rate of growth is higher if agents receive PAYG pensions. ${ }^{14}$ The reason for our finding is that the PAYG system imposes a life-cycle on human wealth that does not otherwise obtain in the standard BY framework. In our specification the PAYG pension puts part of the population, since $(1-\theta)>\pi$, in a lower non-asset income stream. This strengthens the effect of the turnover in human wealth, $[\bar{h}(t, t)-h(t)]>0$, since agents are now born with relatively more human wealth than their older counterparts who face reduced non-asset retirement income. This, in turn, weakens the negative implications that demographic turnover has, in general, on economic growth. Under consumption smoothing, agents respond to the fall in old-age income by increasing saving during their working

\footnotetext{
${ }^{14}$ The sign of (55) is positive since:

$1-\Omega\left(\hat{r}_{g}\right)=\frac{(1-\varepsilon) r}{\varepsilon} d r\left(\pi_{R}\right)(1-\theta-\pi) \frac{1-e^{-\left(\hat{r}_{g}-n\right) u_{R}}}{\hat{r}_{g}-n}>0$.
} 


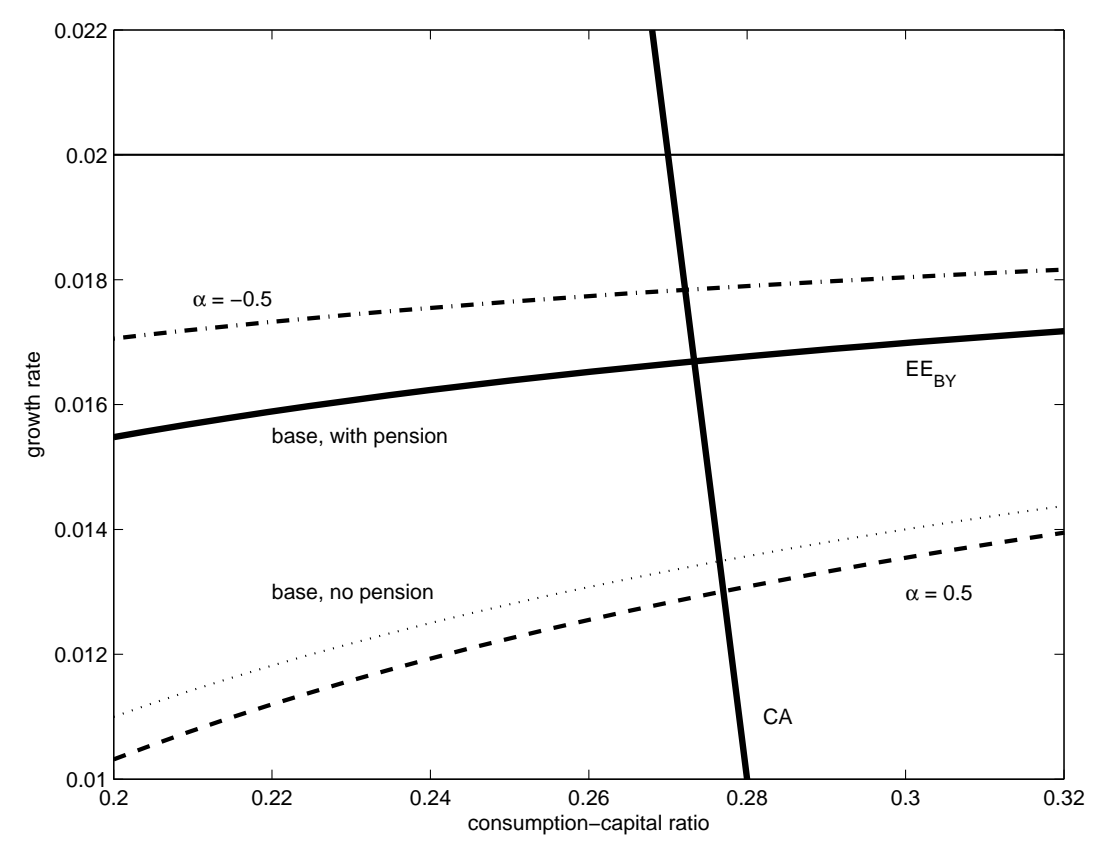

Figure 3: Growth, pensions, and the KUJ effect

lives, which raises the rate of capital accumulation and implies that $\hat{\gamma}^{P}>\hat{\gamma}$. In turn, the expression for $\hat{x}-\hat{x}^{P}$, obtained by linearizing $\Gamma(s)$ from (25) about the equilibrium solved for in (53), equals:

$$
\hat{x}^{P}-\hat{x}=-\frac{1-\Omega\left(\hat{r}_{g}^{P}\right)}{2 \hat{r}_{g}^{P}-(\rho+n)+(1-\varepsilon) Z_{0}}<0,
$$

We depict in Figure 3 the influence of PAYG, where the baseline pension system parameters equal:

$$
d r=0.20, \quad u_{R}=59.73 \text { years }, \quad \theta=0.1, \quad \pi=0.5, \quad r r \equiv \frac{\pi}{1-\theta}=0.45 .
$$

The growth equilibrium with pensions is illustrated by the intersection of the solid $\mathrm{EE}_{\mathrm{BY}}$ and CA relationships: since the solid $\mathrm{EE}_{\mathrm{BY}}$ locus lies entirely above its counterpart in Figure 1 with no pensions (the thinly dotted line), the solution $(\hat{x}, \hat{\gamma})$ under PAYG involves a higher growth rate and a lower consumption-capital ratio. Figure 3 furthermore illustrates how KUJ modifies the role of the pension system. Admiration $(\alpha=-0.5)$ augments the effect of PAYG, raising the growth rate even more, while jealousy $(\alpha=0.5)$ reverses it. Indeed, under our parameterization, jealousy is more 'powerful' than PAYG, since $\hat{\gamma}_{\alpha=0.5}^{P}<\hat{\gamma}_{\alpha=0}$, a result depicted in Figure 3 by comparing the dotted and dashed relationships. 
To determine the effects of an increase in the statutory retirement date on the growth equilibrium, we evaluate (52)-(53) at the solution values $\left(\hat{x}, \hat{r}_{g}\right)$ :

$$
\begin{aligned}
& \Phi\left(\hat{r}_{g}^{P}, \pi, \theta, u_{R}\right) \equiv\left(\hat{r}_{g}^{P}-\rho\right) \cdot\left[\hat{r}_{g}^{P}+(1-\varepsilon) Z_{0}-n\right]-\sigma \Omega\left(\hat{r}_{g}^{P}\right) \equiv 0, \\
& \Gamma\left(\hat{x}^{P}, \pi, \theta, u_{R}\right)=\left(\hat{x}^{P}\right)^{2}-\left[\rho+(1-\varepsilon) Z_{0}-n\right] \hat{x}^{P}-\sigma \Omega\left(\hat{r}_{g}^{P}\right) \equiv 0 .
\end{aligned}
$$

Specifically, we analyze the implications of an increase in the statutory retirement age $u_{R}$ which lowers the dependency ratio under both DB and DC schemes (see (34) above). Differentiation of (58)-(59) with respect to $u_{R}$ yields:

$$
\begin{aligned}
& \left.\frac{\partial \hat{r}_{g}^{P}}{\partial u_{R}}\right|_{i}=-\left.\frac{\partial \hat{\gamma}^{P}}{\partial u_{R}}\right|_{i}=-\frac{\left[\partial \Phi\left(\hat{r}_{g}^{P}, \pi, \theta, u_{R}\right) / \partial u_{R}\right]_{i}}{\partial \Phi\left(\hat{r}_{g}^{P}, \pi, \theta, u_{R}\right) / \partial \hat{r}_{g}^{P}}, \\
& \left.\frac{\partial \hat{x}^{P}}{\partial u_{R}}\right|_{i}=-\frac{\left[\partial \Gamma\left(\hat{x}^{P}, \pi, \theta, u_{R}\right) / \partial u_{R}\right]_{i}}{\partial \Gamma\left(\hat{x}^{P}, \pi, \theta, u_{R}\right) / \partial \hat{x}^{P}},
\end{aligned}
$$

for $i=\mathrm{DB}$ or $i=\mathrm{DC}$. We can show that a higher retirement age has an ambiguous effect on growth and the consumption-capital ratio under both schemes. Nevertheless, we can identify the distinct implications of a rise in $u_{R}$. An increase in $u_{R}$ raises labor supply, lowering the wage $w(t)$, which, in turn, shrinks the human wealth gap, $[\bar{h}(t, t)-h(t)]$, and lowers $\hat{\gamma}^{P}$. On the other hand, a later statutory retirement age means that a larger fraction of the population participates in the labor force. This increases generational turnover and raises $\hat{\gamma}^{P}$. The latter effect is augmented by the fact that the contribution rate $\theta$ paid by the active part of the population falls to maintain PAYG budget balance under DB.

The general implications of a rise in $u_{R}$ under DC are similar to those under DB, with the important exception that in the DC case the pay-out rate $\pi$ received by retired workers increases under the closure rule. This mitigates against the effect of a longer working life and tends to reduce the human wealth gap, $[\bar{h}(t, t)-h(t)]$. For our parameterization, for which the mandatory retirement age rises from 59.73 to 65 years, the negative effect on growth of the rise in $u_{R}$ dominates, implying a decline in $\mathrm{EE}_{\mathrm{BY}}$ in Figure 4, whether or not the PAYG system is DB or DC. Observe in Figure 4, however, that the fall in growth is significantly greater, due to the increase in $\pi$ in the DC case, compared to the system under DB. Our results suggest, then, that pension reform is more effective at maintaining growth under a DB system.

\section{Conclusions}

A key limitation of the RA model compared to its OLG counterpart is its assumption of agent homogeneity. The effects of demographic evolution, of course, cannot be addressed in the 


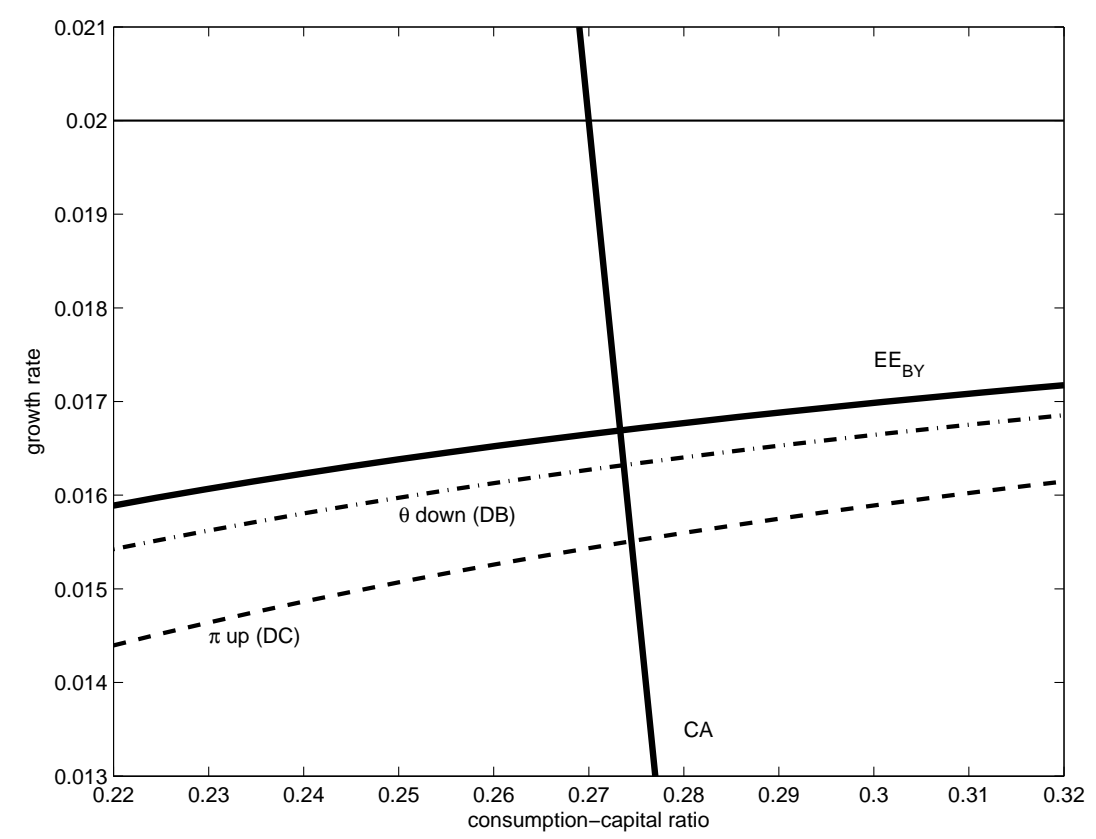

Figure 4: Growth and later retirement

RA setting. In contrast, the BY version of the OLG framework is particularly suited, due to its convenient dynamic structure, to study the macroeconomic implications of demographic change. In this paper we seek to investigate the implications of intergenerational turnover for endogenous growth by extending our BY framework - featuring a well-defined demography and consumption externalities - to the standard $A K$ growth model. We show that the BY model extends naturally to the $A K$ setting and provides a new avenue with which to study the relationship between demography and economic growth. Among our findings, we determine that the balanced growth rate, due to intergenerational turnover, is lower in the BY framework compared to the basic AK model. Regarding demographic shocks, we show that a fall in fertility and a decline in mortality - both characteristic of modern, industrialized societies - lead to a rise in the balanced growth rate. A greater degree of status preference, in contrast, leads to a decline in economic growth, since the effects of intergenerational turnover become more pronounced.

In the second part of the paper we modify the BY model to incorporate a policy intervention - a PAYG pension system - that directly impacts on the life-cycle return to human wealth. We find that a PAYG system increases the balanced growth rate compared to an economy that lacks one. The reason is that PAYG pensions impose an exogenous retirement date and an old-age non-asset income stream that is lower than that of the active part of the pop- 
ulation. This creates an intergenerational turnover effect in human wealth, with newborns possessing more of this type of wealth than average. This, in turn, acts as a countervailing influence to the fact that newborns, compared to older population cohorts, have no financial wealth. With respect to changes in the parameters of the pension system, we show, using a plausible numerical parameterization of the model, that an increase in the statutory retirement date lowers balanced growth under both DB and DC schemes, although the decline in growth is much less under DB.

\section{Appendix A: Derivation of equations (16)-(17)}

To calculate the expression for $\dot{c}(t) / c(t)$ in (16), we use Leibnitz's Rule to differentiate $c(t)$, stated in (13), with respect to $t$ :

$$
\begin{aligned}
\dot{c}(t) & \equiv l(t, t) \bar{c}(t, t)+\int_{-\infty}^{t} l(v, t) \dot{\bar{c}}(v, t) d v+\int_{-\infty}^{t} \dot{l}(v, t) \bar{c}(v, t) d v \\
& =\eta \bar{c}(t, t)+\int_{-\infty}^{t} l(v, t) \dot{\bar{c}}(v, t) d v-\eta \int_{-\infty}^{t} l(v, t) \bar{c}(v, t) d v \\
& =\int_{-\infty}^{t} l(v, t) \dot{\bar{c}}(v, t) d v-\eta[c(t)-\bar{c}(t, t)],
\end{aligned}
$$

where we use (12) and (13) to obtain the second and third equalities of (A.1). The next step, using the definition of $\bar{x}(v, \tau)$ in (6), is to substitute for $\dot{\bar{c}}(v, t)$ in the first term of (A.1). Since $\dot{\bar{c}}(v, t) \equiv(1-\alpha) \dot{\bar{x}}(v, t)+\alpha \dot{c}(t)$, this yields:

$$
\begin{aligned}
\dot{c}(t) & =(1-\alpha) \int_{-\infty}^{t} l(v, t) \dot{\bar{x}}(v, t) d v+\alpha \int_{-\infty}^{t} l(v, t) \dot{c}(t) d v-\eta[c(t)-\bar{c}(t, t)] \\
& =(1-\alpha)[r(t)-\rho] \int_{-\infty}^{t} l(v, t) \bar{x}(v, t) d v+\alpha \dot{c}(t) \int_{-\infty}^{t} l(v, t) d v-\eta[c(t)-\bar{c}(t, t)],
\end{aligned}
$$

where we substitute for $\dot{\bar{x}}(v, t)=(r-\rho) \bar{x}(v, t)$ using (8) to obtain the second equality of (A.2). Analogous to the definition of average consumption, $x(t) \equiv \int_{-\infty}^{t} l(v, t) \bar{x}(v, t) d v$. Furthermore, since cohort weights sum-up to unity, $\int_{-\infty}^{t} l(v, t) d v \equiv 1$, and $x(t)=c(t)$ holds by definition, we can rewrite (A.2) to obtain the aggregate Euler equation:

$$
\dot{c}(t)=[r(t)-\rho] c(t)-\frac{\eta}{1-\alpha} \cdot[c(t)-\bar{c}(t, t)],
$$

where $[c(t)-\bar{c}(t, t)]$ corresponds to intergenerational turnover in consumption. To convert (A.3) into the expression (16) in the main text, we evaluate (11) at $v=t$ to find:

$$
\bar{c}(t, t)=(\rho+\beta) h(t)+\alpha[c(t)-(\rho+\beta) \Gamma(t)],
$$

where $\bar{a}(t, t)=0$, since newborns only possess human wealth. In turn, aggregating (11) over cohorts implies:

$$
c(t)=(\rho+\beta)[a(t)+h(t)]+\alpha[c(t)-(\rho+\beta) \Gamma(t)] .
$$


Taking the difference between (A.5) and (A.4), we obtain $[c(t)-\bar{c}(t, t)]=(\rho+\beta) a(t)$ so that (A.3) reduces to (16):

$$
\frac{\dot{c}(t)}{c(t)}=r-\rho-\frac{\eta(\rho+\beta)}{1-\alpha} \frac{k(t)}{c(t)}
$$

where we have used $a(t)=k(t)$. To derive (17), we differentiate $a(t)$ stated in (13) with respect to $t$ to find:

$$
\dot{a}(t)=-\eta \int_{-\infty}^{t} l(v, t) \bar{a}(v, t) d v+\int_{-\infty}^{t} l(v, t) \dot{\bar{a}}(v, \tau) d v,
$$

where we again use the fact that $\bar{a}(t, t)=0$ and substitute for $\dot{l}(v, t)=-\eta l(v, t)$ to obtain (A.6). Substituting for $\dot{\bar{a}}(v, \tau)$ from the budget identity (7) in (A.6), we obtain:

$$
\begin{aligned}
\dot{a}(t)= & -\eta \int_{-\infty}^{t} l(v, t) \bar{a}(v, t) d v \\
& +\int_{-\infty}^{t} l(v, t)[(r+\beta) \bar{a}(v, \tau)+w(\tau)-\bar{c}(v, \tau)] d v \\
= & (r-n) a(t)+w(t)-c(t),
\end{aligned}
$$

where $n \equiv \eta-\beta$. Finally, the fact that physical capital is the only form of savings, i.e., $a(t)=k(t)$, means that (A.7) is equivalent to the market clearing relationship (17):

$$
\dot{k}(t)=[r-n] k(t)+w(t)-c(t) .
$$

\section{Appendix B: Conditions on steady-state profiles}

\section{B.1 Condition for $\bar{k}(0, t)>0$}

We begin by evaluating $(7)$ at $\bar{a}(v, t) \equiv \bar{k}(v, t)$ and substituting for $\hat{w}(t)=\hat{w}_{0} e^{\hat{\gamma} t}$. Together with (15), this yields:

$$
\begin{aligned}
& \dot{\bar{k}}(v, t)=(r+\beta) \bar{k}(v, t)+\hat{w}_{0} e^{\hat{\gamma} t}-\bar{c}(v, t) \\
& \bar{c}(v, t)-\hat{c}(t)=(\rho+\beta)[\bar{k}(v, t)-\hat{k}(t)] .
\end{aligned}
$$

Combining these expressions, we obtain the following differential equation in $\dot{\bar{k}}(v, t)$ :

$$
\dot{\bar{k}}(v, t)=(r-\rho) \bar{k}(v, t)+(\eta+\rho+\hat{\gamma}-r) \hat{k}_{0} e^{\hat{\gamma} t} .
$$

Solving (B.3) for $v=0$, subject to $\bar{k}(0,0)=0$, gives:

$$
\bar{k}(0, t)=\frac{\rho+\eta+\hat{\gamma}-r}{r-\hat{\gamma}-\rho} \cdot \hat{k}(t) \cdot\left[e^{(r-\hat{\gamma}-\rho) t}-1\right]
$$

which yields the following conditions for $\bar{k}(0, t)>0$ :

$$
\text { (i) } r-\hat{\gamma} \equiv \hat{r}_{g}>\rho ; \quad \text { (ii) } r-\hat{\gamma} \equiv \hat{r}_{g}<\rho+\eta .
$$


B.2 Condition for $\bar{c}(0, t)>0$

Using the solutions $\hat{k}(t)=\hat{k}_{0} e^{\hat{\gamma} t}, \hat{w}(t)=\hat{w}_{0} e^{\hat{\gamma} t}$, and $\hat{c}(t)=\hat{c}_{0} e^{\hat{\gamma} t}$ along the growth path, we can rewrite the Euler equation (16) and the market clearing condition (17) as:

$$
\begin{aligned}
& (1-\alpha)(r-\rho-\hat{\gamma}) \hat{c}_{0}=\eta(\rho+\beta) \hat{k}_{0} \\
& {[r-\hat{\gamma}-(\eta-\beta)] \hat{k}_{0}=\hat{c}_{0}-\hat{w}_{0},}
\end{aligned}
$$

Evaluating (B.2) at $v=0$ and substituting for $\hat{c}(t)$ employing (B.6), newborn consumption equals:

$$
\bar{c}(0, t)=\frac{\eta(\rho+\beta) \hat{k}(t)}{(1-\alpha)(r-\rho-\hat{\gamma})}+(\rho+\beta)[\bar{k}(0, t)-\hat{k}(t)] .
$$

Employing the solution (B.4) for $\bar{k}(0, t)$, we substitute $[\bar{k}(0, t)-\hat{k}(t)]$ into (B.7) and, after simplifying, we obtain the expression for $\bar{c}(0, t)$ in terms of $\hat{k}(t)$ :

$$
\bar{c}(0, t)=\frac{(\rho+\beta) \hat{k}(t)}{r-\rho-\hat{\gamma}}\left[(\eta+\rho+\hat{\gamma}-r) e^{(r-\hat{\gamma}-\rho) t}+\frac{\alpha \eta}{1-\alpha}\right] .
$$

A feasible solution for newborn consumption, i.e., $\bar{c}(0, t)>0$, requires:

$$
(\eta+\rho+\hat{\gamma}-r)+\frac{\alpha \eta}{1-\alpha}>0,
$$

a condition automatically satisfied for $0 \leq \alpha<1$. If, instead, $\alpha<0$, then we must determine a lower bound on the status parameter so that:

$$
r-\hat{\gamma} \equiv \hat{r}_{g}<\eta+\rho+\frac{\alpha \eta}{1-\alpha}
$$

a task we perform now.

\section{B.3 Upper and Lower Bounds on $\alpha$}

We first derive the upper bound on the status parameter $\alpha$. To do so, we use the polynomial $\Phi(s)$ stated in (24) to prove $\hat{r}_{g}<\rho+\eta \equiv r_{g 1}$ stated in (B.5). This holds if $\Phi\left(r_{g 1}\right)>0$. Evaluating $\Phi\left(r_{g 1}\right)$, we find:

$$
\Phi\left(r_{g 1}\right)=\eta \cdot\left[(1-\varepsilon) Z_{0}-(\rho+\beta) \frac{\alpha}{1-\alpha}\right] .
$$

For $\alpha<0, \Phi\left(r_{g 1}\right)>0$ is automatically satisfied. For $\alpha>0$, the following upper bound obtains:

$$
\frac{\alpha}{1-\alpha}<\frac{(1-\varepsilon) Z_{0}}{\rho+\beta}
$$


To determine the lower bound on $\alpha$, we prove $\hat{r}_{g}<\rho+\eta+\frac{\alpha \eta}{1-\alpha} \equiv r_{g 2}$ given in (B.10) for $\alpha<0$. Evaluating $\Phi(s)$ at $s=r_{g 2}$, we show:

$$
\Phi\left(r_{g 2}\right)=\frac{\eta}{1-\alpha} \cdot\left[\frac{\alpha \eta}{1-\alpha}+(1-\varepsilon) Z_{0}\right] .
$$

The result $\Phi\left(r_{g 2}\right)>0$ holds as long as:

$$
\frac{\alpha}{1-\alpha}>-\frac{(1-\varepsilon) Z_{0}}{\eta}
$$

Combining (B.12) and (B.14), we state the feasible range for $\alpha$ :

$$
-\frac{(1-\varepsilon) Z_{0}}{\eta}<\frac{\alpha}{1-\alpha}<\frac{(1-\varepsilon) Z_{0}}{\rho+\beta} .
$$

\section{Appendix C: Derivation of (46) and (47)}

To find the expression for (46), we first solve for $h(t)$ by substituting (43)-(44) that describe, respectively, human wealth for workers and retirees, into (35). This yields:

$$
\begin{aligned}
h(t)= & \frac{w(t)}{r_{g}+\beta} \cdot\left[(1-\theta)\left[1-e^{-\eta u_{R}}\right]+\eta[\pi-(1-\theta)] e^{-\eta u_{R}} \cdot \frac{1-e^{-\left(r_{g}-n\right) u_{R}}}{r_{g}-n}\right. \\
& \left.+\pi e^{-\eta u_{R}}\right] .
\end{aligned}
$$

Employing the PAYG balanced-budget rule, we can simplify (C.1) and obtain:

$$
h(t)=\frac{w(t)}{r_{g}+\beta} \cdot\left[1-e^{-\eta u_{R}}-\eta e^{-\beta u_{R}}(1-\theta-\pi) \cdot \frac{e^{-n u_{R}}-e^{-r_{g} u_{R}}}{r_{g}-n}\right] .
$$

Human wealth represents the present discounted value of wages, adjusted by the features of the PAYG system and demographic parameters. Evaluating (43) at $v=t$, we can show that newborn agents begin life with:

$$
\bar{h}(t, t)=\frac{w(t)}{r_{g}+\beta} \cdot\left[1-e^{-\eta u_{R}}+(1-\theta-\pi) e^{-\beta u_{R}} \cdot\left[e^{-n u_{R}}-e^{-r_{g} u_{R}}\right]\right] .
$$

Combining (C.2)-(C.3), the turnover in human wealth thus equals:

$$
\bar{h}(t, t)-h(t)=w(t) e^{-\beta u_{R}}(1-\theta-\pi) \cdot \frac{e^{-n u_{R}}-e^{-r_{g} u_{R}}}{r_{g}-n}>0,
$$

To derive (47) for the PAYG case, we modify (A.6) to reflect the working and retired phases of life:

$$
\dot{a}(t)=-\eta \int_{-\infty}^{t} l(v, t) \bar{a}(v, t) d v+\int_{t-u_{R}}^{t} l(v, t) \dot{\bar{a}}(v, \tau) d v+\int_{-\infty}^{t-u_{R}} l(v, t) \dot{\bar{a}}(v, \tau) d v .
$$


Using (41) and (31) to substitute for $\dot{a}(t)$ in (C.5), we obtain:

$$
\dot{a}(t)=(r-n) a(t)+(1-\theta) w(t) \int_{t-u_{R}}^{t} l(v, t) d v+\pi w(t) \int_{-\infty}^{t-u_{R}} l(v, t) d v-c(t),
$$

where we use the definitions of average consumption and asset holdings in (13). Substituting for the cohort weights $l(v, t)=\eta e^{\eta t}, t \geq v$, we evaluate (C.6) as:

$$
\dot{a}(t)=(r-n) a(t)+(1-\theta) w(t)\left(1-e^{-\eta u_{R}}\right)+\pi w(t) e^{-\eta u_{R}}-c(t) .
$$

To simplify (C.7) and solve for the market clearing condition, we impose the PAYG budget constraint $\theta \cdot\left[1-e^{-\eta u_{R}}\right]=\pi \cdot e^{-\eta u_{R}}$ and $a(t) \equiv k(t)$ and obtain:

$$
\dot{k}(t)=[r-n] k(t)+w(t)\left(1-e^{-\eta u_{R}}\right)-c(t) .
$$

\section{Appendix D: PAYG and the welfare of newborns}

Using (43), we can ask the whether the PAYG system imposes a net burden on newborns. The scenario we consider is the following: assume that newborns, whether or not they are participants in the PAYG scheme, retire exogenously at age $u_{R}$. If the newborn agent pays no contributions and receives no future benefits $(\theta=\pi=0)$, his human wealth is:

$$
[\bar{h}(t, t)]_{N}=\frac{w(t)}{r_{g}+\beta}\left[1-e^{-\left(r_{g}+\beta\right) u_{R}}\right] .
$$

In contrast, if the agent is within the pension system, his human wealth corresponds to:

$$
[\bar{h}(t, t)]_{P}=[\bar{h}(t, t)]_{N}-\frac{w(t)}{r_{g}+\beta}\left\{\theta\left[1-e^{-\left(r_{g}+\beta\right) u_{R}}\right]-\pi e^{-\left(r_{g}+\beta\right) u_{R}}\right\} .
$$

Substituting for the balance-budget rule $0=-\theta\left[1-e^{-\eta u_{R}}\right]+\pi e^{-\eta u_{R}}$, (D.1) simplifies to:

$$
[\bar{h}(t, t)]_{P}-[\bar{h}(t, t)]_{N}=-\frac{w(t)}{r_{g}+\beta} \cdot(\theta+\pi) e^{-\beta u_{R}}\left[e^{-n u_{R}}-e^{-r_{g} u_{R}}\right]
$$

The question whether PAYG pensions reduce or augment $\bar{h}(t, t)$ dependes on the sign of the term $\left[e^{-n u_{R}}-e^{-r_{g} u_{R}}\right]$, which, in turn, depends on $r_{g}$ relative to $n$. If the well-known Aaron condition holds, i.e., $r_{g}>n$, then the sign of (D.3) is negative and the PAYG system reduces human wealth at birth.

\section{References}

Alvarez-Cuadrado, F., Monteiro, G., and Turnovsky, S. J., 2004. Habit formation, catching up with the Joneses, and economic growth. Journal of Economic Growth 9, 47-80. 
Barro, R.J., 1990. Government spending in a simple model of endogenous growth. Journal of Political Economy 98, S103-S125.

Bettendorf, L.J.H. and Heijdra, B.J., 2006 Population aging and pension reform in a small open economy with non-traded goods. Journal of Economic Dynamics and Control 30, 2389-2424.

Blanchard, O. J., 1985. Debt, deficits, and finite horizons. Journal of Political Economy 91, 589-610.

Dupor, B. and Liu, W. F., 2003. Jealousy and equilibrium overconsumption. American Economic Review, 423-428.

Fisher, W. H. and Heijdra, B. J., 2008. Keeping up with the ageing Joneses. Journal of Economic Dynamics and Control, forthcoming.

Heijdra, B. J. and Ligthart, J. E., 2006. The macroeconomic dynamics of demographic shocks. Macroeconomic Dynamics 10, 349-370.

Liu, W. F. and Turnovsky, S. J., 2005. Consumption externalities, production externalities, and the accumulation of capital. Journal of Public Economics 89, 1097-1129.

Rebelo, S., 1991. Long-run policy analysis and long-run growth. Journal of Political Economy $99,500-521$.

Romer, P. M., 1986. Increasing returns and long-run growth. Journal of Political Economy 94, 1002-1037.

Romer, P.M., 1989. Capital accumulation in the theory of long-run growth. In Barro, R.J. ed., Modern Business Cycle Theory, 51-127, Basil Blackwell, Oxford.

Saint-Paul, G., 1992. Fiscal policy in an endogenous growth model. Quarterly Journal of Economics 107, 1243-1259.

Turnovsky, S.J. and Monteiro, G., 2007. Consumption externalties, production externalities, and efficient capital accumulation under time non-separable preferences. European Economic Review 51, 479-504.

Yaari, M. E., 1965. Uncertain lifetime, life insurance, and the theory of the consumer. Review of Economic Studies 32, 137-150. 
Authors: Walter H. Fisher, Ben J. Heijdra

Title: Growth and the Ageing Joneses

Reihe Ökonomie / Economics Series 230

Editor: Robert M. Kunst (Econometrics)

Associate Editors: Walter Fisher (Macroeconomics), Klaus Ritzberger (Microeconomics)

ISSN: 1605-7996

(C) 2008 by the Department of Economics and Finance, Institute for Advanced Studies (IHS),

Stumpergasse 56, A-1060 Vienna • ㅍㅛㅛ +43 1 59991-0 • Fax +43 1 59991-555 • http://www.ihs.ac.at 
ISSN: 1605-7996 